\title{
Quantum computing thanks to Bianchi groups
}

\author{
Michel Planat ${ }^{1, *}$ \\ ${ }^{1}$ Université de Bourgogne/Franche Comté, Institut FEMTO-ST UMR 6174, 15 B Avenue des Mont- \\ boucons, F-25044 Besançon, France
}

\begin{abstract}
It has been shown that the concept of a magic state (in universal quantum computing: uqc) and that of a minimal informationally complete positive operator valued measure: MIC-POVMs (in quantum measurements) are in good agreement when such a magic state is selected in the set of nonstabilizer eigenstates of permutation gates with the Pauli group acting on it [1]. Further work observed that most found low-dimensional MICs may be built from subgroups of the modular group $P S L(2, \mathbb{Z})[2]$ and that this can be understood from the picture of the trefoil knot and related 3-manifolds [3]. Here one concentrates on Bianchi groups $\operatorname{PS} L\left(2, O_{d}\right)$ (with $O_{d}$ the integer ring over the imaginary quadratic field) whose torsion-free subgroups define the appropriate knots and links leading to MICs and the related uqc. One finds a chain of Bianchi congruence $n$-cusped links playing a significant role [4].
\end{abstract}

\section{Introduction}

A Bianchi group $\Gamma_{k}=\operatorname{PS} L\left(2, O_{k}\right)<\operatorname{PS} L(2, \mathbb{C})$ acts as a subset of orientation-preserving isometries of 3-dimensional hyperbolic space $\mathbb{H}_{3}$ with $O_{k}$ the ring of integers of the imaginary quadratic field $\mathcal{I}=\mathbb{Q}(\sqrt{-k})$. The quotient space 3-orbifold $P S L\left(2, O_{k}\right) \backslash \mathbb{H}_{3}$ has a set of cusps in bijection with the class group of $\mathcal{I}$ [5-7]. A torsion-free subgroup $\Gamma_{k}(l)$ of index $l$ of $\Gamma_{k}$ is the fundamental group $\pi_{1}$ of a 3-manifold defined by a link such as the figure-of-eight knot [with $\Gamma_{-3}(12)$ ], the Whitehead link [with $\Gamma_{-1}(12)$ ] or the Borromean rings [with $\left.\Gamma_{-1}(24)\right]$. The fundamental group of the knot complement in the three sphere $S_{3}$ also called the knot group, or similarly the link group [5,6], was used to construct appropriate $d$-dimensional fiducial states for universal quantum computing (uqc) [3] thanks to some of their degree $d$ coverings.

In this paper, one starts by upgrading these models of uqc by using other torsion-free subgroups of Bianchi groups and their corresponding 3-manifold such as the Bergé manifold that comes from the Bergé link $L 6 a 2$ [with $\Gamma_{-3}(24)$ ] or the so-called magic manifold that comes from the link $L 6 a 5$ [with $\Gamma_{-7}(6)$ ]. The latter link is a small congruence link and belongs to a chain of eight links starting with Thurston's eight-cusped congruence link [with $\Gamma_{-3}$ and ideal $\langle(5+\sqrt{-3}) / 2\rangle[8,9]$ and ending with the Whitehead link and then the figureeight knot.

In Sec. 2, one reminds the permutation based model of universal quantum computing developed by the authors and its relationship to minimal informationally complete POVMs or MICs [1-3]. In Sec. 3, important small index torsion-free subgroups of Bianchi groups and the corresponding substructure of 3-manifolds are derived. In Sec. 4, one specializes in

\footnotetext{
*e-mail: michel.planat@femto-st.fr
} 
the connection of the aforementioned Bianchi subgroups to our version of uqc. One features on a remarkable chain of $n$-cusped $n$-links, $n=8 . .1$ obtained thanks to $( \pm 1,1)$-slope Dehn filling starting with congruence Thurston's link. Their possible role for uqc and the relevant 3-manifolds is discussed.

\section{Minimal informationally complete POVMs with permutations}

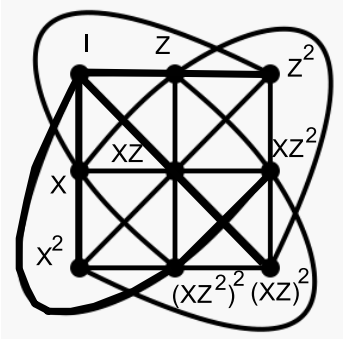

The Hesse SIC for qutrits fiducials : $(0,1,1)$ or $(0,1,-1)$

(a)

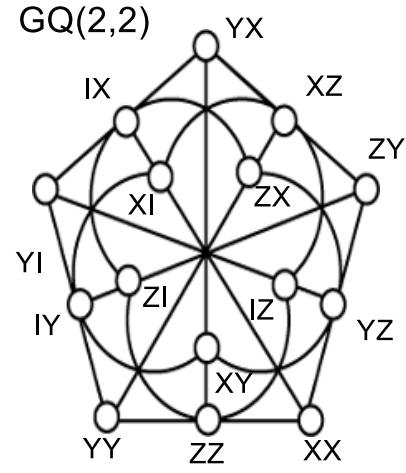

The two-qubit MIC fiducial : e.g. $(0,1,-\omega 6, \omega 6-1)$

(b)

Figure 1. Geometrical structure of low dimensional MICs: (a) the qutrit Hesse SIC, (b) the two-qubit MIC that is the generalized quadrangle of order two $G Q(2,2)$. The coordinates on each diagram are the $d$-dimensional Pauli operators that act on the fiducial state, as shown.

In our approach [1, 2], minimal informationally complete POVMs (MICs) are derived from appropriate fiducial states under the action of the (generalized) Pauli group. The fiducial states also allow to perform universal quantum computation [10].

A POVM is a collection of positive semi-definite operators $\left\{E_{1}, \ldots, E_{m}\right\}$ that sum to the identity. In the measurement of a state $\rho$, the $i$-th outcome is obtained with a probability given by the Born rule $p(i)=\operatorname{tr}\left(\rho E_{i}\right)$. For a minimal IC-POVM, one needs $d^{2}$ onedimensional projectors $\Pi_{i}=\left|\psi_{i}\right\rangle\left\langle\psi_{i}\right|$, with $\Pi_{i}=d E_{i}$, such that the rank of the Gram matrix with elements $\operatorname{tr}\left(\Pi_{i} \Pi_{j}\right)$, is precisely $d^{2}$. A SIC-POVM (the $S$ means symmetric) obeys the relation $\left|\left\langle\psi_{i} \mid \psi_{j}\right\rangle\right|^{2}=\operatorname{tr}\left(\Pi_{i} \Pi_{j}\right)=\frac{d \delta_{i j}+1}{d+1}$, that allows the explicit recovery of the density matrix as in [11, eq. (29)].

New MICs (i.e. whose rank of the Gram matrix is $d^{2}$ ) and with Hermitian angles $\left|\left\langle\psi_{i} \mid \psi_{j}\right\rangle\right|_{i \neq j} \in A=\left\{a_{1}, \ldots, a_{l}\right\}$ have been discovered [2]. A SIC is equiangular with $|A|=1$ and $a_{1}=\frac{1}{\sqrt{d+1}}$. The states encountered are considered to live in a cyclotomic field $\mathbb{F}=\mathbb{Q}\left[\exp \left(\frac{2 i \pi}{n}\right)\right]$, with $n=\operatorname{GCD}(d, r)$, the greatest common divisor of $d$ and $r$, for some $r$. The Hermitian angle is defined as $\left|\left\langle\psi_{i} \mid \psi_{j}\right\rangle\right|_{i \neq j}=\left\|\left(\psi_{i}, \psi_{j}\right)\right\|^{\frac{1}{\operatorname{deg}}}$, where $\|$.$\| means the field norm$ of the pair $\left(\psi_{i}, \psi_{j}\right)$ in $\mathbb{F}$ and deg is the degree of the extension $\mathbb{F}$ over the rational field $\mathbb{Q}$ [1].

The fiducial states for SIC-POVMs are quite difficult to derive and seem to follow from algebraic number theory [12]. Except for $d=3$, the MICs derived from permutation groups 
are not symmetric and most of them can be recovered thanks to subgroups of index $d$ of the modular group $\Gamma$ [2, Table 1]. For instance, for $d=3$, the action of a magic state of type $(0,1, \pm 1)$ results into the Hesse SIC shown in Fig 1a, arising from the congruence subgroup $\Gamma_{0}(2)$ of $\Gamma$. For $d=4$, the action of the two-qubit Pauli group on the magic/fiducial state of type $\left(0,1,-\omega_{6}, \omega_{6}-1\right)$ with $\omega_{6}=\exp \left(\frac{2 i \pi}{6}\right)$ results into a MIC whose geometry of triple products of projectors $\Pi_{i}$, arising from the the congruence subgroup $\Gamma_{0}(3)$ of $\Gamma$, turns out to correspond to the commutation graph of Pauli operators, see Fig. $1 \mathrm{~b}$ and [2, Fig. 2c]. For $d=5$, the congruence subgroup $5 A^{0}$ of $\Gamma$ is used to get a MIC whose geometry consists of copies of the Petersen graph (see [2, Fig. 3c]. For $d=6$, all five congruence subgroups $\Gamma^{\prime}$, $\Gamma(2), 3 C^{0}, \Gamma_{0}(4)$ or $\Gamma_{0}(5)$ point out the geometry of Borromean rings (see

The modular group $\Gamma$ first served as a motivation for investigating the trefoil knot manifold $3_{1}$ in relation to uqc and the corresponding MICs, then the uqc problem was put in the wider frame of Poincaré conjecture, the Thurston's geometrization conjecture and the related 3manifolds. E.g. MICs may follow from hyperbolic or Seifert 3-manifolds as shown in Tables 2 to 5 of [3]. A further step is obtained here by restricting our choice of 3-manifolds to low degree coverings of Bianchi subgroups. This is a quite natural procedure to base our uqc models on Bianchi groups $\Gamma_{k}$ defined over the ring $O_{k}$ of imaginary quadratic integers [13] since they are a natural generalization of the modular group $\Gamma$.

\section{Torsion-free subgroups of Bianchi groups.}

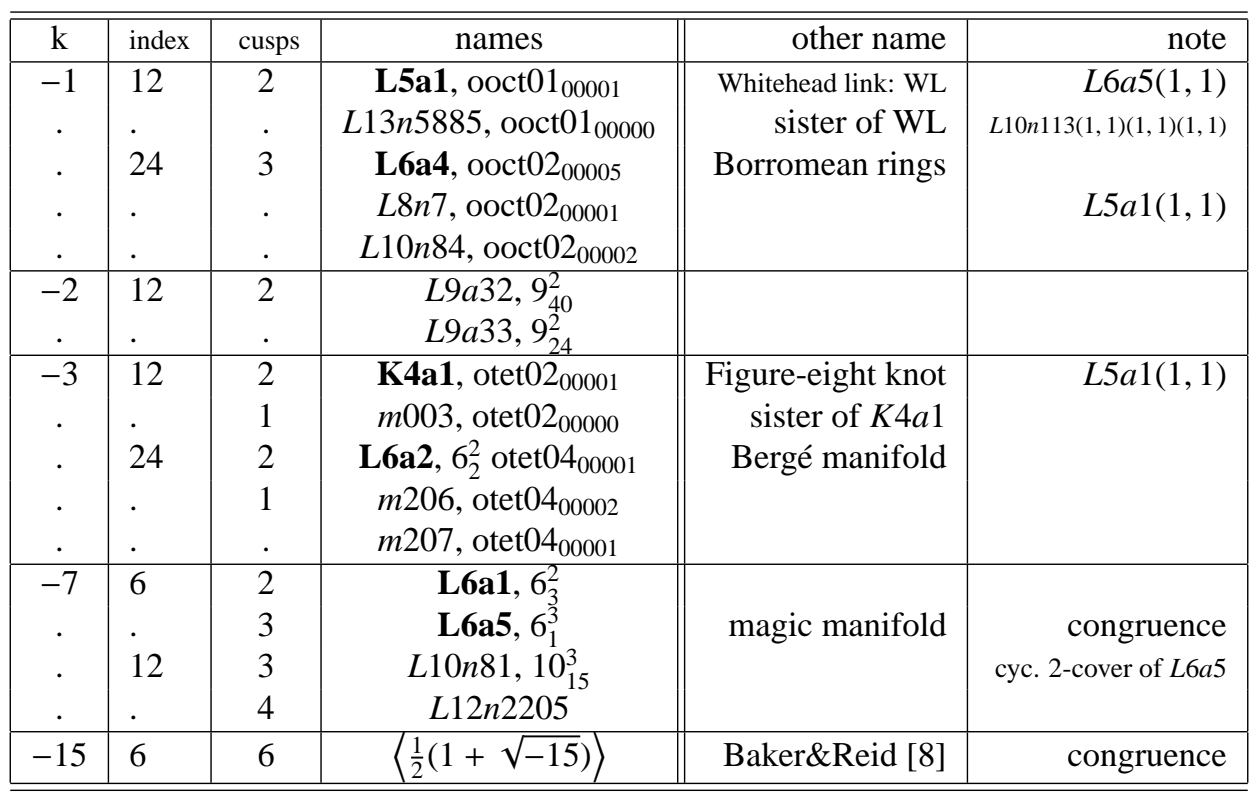

Table 1. Low index torsion-free subgroups of Bianchi groups $\Gamma_{k}=P S L\left(2, O_{k}\right), k=-1,-2,-3,-7$, their names in SnapPy in column 4 and their popular name in column 5, when known. The last column shows a connection to Dehn filling of slope $(1,1)$ of the corresponding manifold. 'Bold' manifolds are investigated here in relation to the existence of MICs from their subgroups of finite index.

Table 1 provides a short list of low index torsion-free subgroups of Bianchi groups $\Gamma_{k}$, $k \in\{-1,-2,-3,-7,-15\}$ [13] obtained from the software Magma [14] and their identification as fundamental groups of 3-manifolds obtained from the software SnapPy [15]. In particular, 
one recovers the Whitehead link and the Borromean rings $(k=-1$ with index $l=12$ and 24 , respectively) as well as the figure-eight knot $(k=-3$ with index 12) whose relationship to uqc was explored in [3]. Other links of importance in our paper are the link $L 6 a 1$, the Bergé link $L 6 a 2$ and the magic link $L 6 a 5$ whose connections to uqc is summarized in Table 2.

According to [16], a $d$-fold covering of the fundamental group $\pi_{1}(M)$ of a 3-manifold $M$ is uniquely determined by the conjugacy class of a subgroup of index $d$ of $\pi_{1}$. To recognize the $d$-covering from the conjugacy class and conversely, one makes use of the cardinality signature of subgroups of $\pi_{1}(M)$ denoted $\eta_{d}\left(\pi_{1}(M)\right), d=1 . . d$ max and one identifies $\eta_{d}\left(\pi_{1}(M)\right)$ both in a particular $d$-covering of $M$ (with SnapPy) and in the representative of a particular conjugacy class of subgroups of $\pi_{1}(M)$ (with Magma).

\section{A Bianchi factory for quantum computing}

First one provides a reminder about the concept of Dehn filling that will be useful in Sect. 4.2. Let us start with a Lens space $L(p, q)$ that is 3-manifold obtained by gluing the boundaries of two solid tori together, so that the meridian of the first solid torus goes to a $(p, q)$-curve on the second solid torus [where a $(p, q)$-curve wraps around the longitude $p$ times and around the meridian $q$ times]. Then we generalize this concept to a knot exterior, i.e. the complement of an open solid torus knotted like the knot. One glues a solid torus so that its meridian curve goes to a $(p, q)$-curve on the torus boundary of the knot exterior, an operation called Dehn surgery [6, p. 275],[7, p 259],[17]. According to Lickorish's theorem, every closed, orientable, connected 3-manifold is obtained by performing Dehn surgery on a link in the 3-sphere.

One shows below how the Bianchi subgroups are related to uqc and the related MICs and how useful is Dehn filling in our context.

\subsection{Universal quantum computing from the Bianchi factory}

In this subsection, one specializes on uqc based on the qutrit Hesse SIC (shown in Fig. 1a) and on the two-qubit geometry of the generalized quadrangle $G Q(2,2)$ (shown in Fig. 1b) obtained with the 'magic' link L6a5. The qutrit uqc follows from a link called 'L14n63788' with Poincaré polyhedron shown in Fig. 2a and the two-qubit uqc follows from a 3-manifold with Poincaré polyhedron shown in Fig $2 b$. The former case corresponds to the 3-fold irregular covering the fundamental group $\pi_{1}(L 6 a 5)$ whose 3 -manifold has first homology $\mathbb{Z}^{\oplus 5}, 5$ cusps and volume $\approx 16.00$. The latter case corresponds to the 4-fold irregular covering whose 3 -manifold has first homology $\mathbb{Z} / 2 \oplus \mathbb{Z}^{\oplus 4}, 4$ cusps and volume $\approx 21.34$.

For the other links investigated in Table 2 one proceeds in the same way.

\subsection{Congruence links in the Bianchi factory}

As announced earlier, there is an interesting sequence of links starting at the Thurston's link and ending at the figure-eight knot that one obtains by applying $( \pm 1,1)$-slope Dehn fillings. The Dehn fillings of last five links called $M_{i}, i=5 . .1$ were studied in [18]. Observe that $M_{3}$ is the magic link $L 6 a 4, M_{4}$ is the 4-link $L 8 n 7$ and $M_{5}$ is the 5-link L10n113 of Table 2. The full sequence is

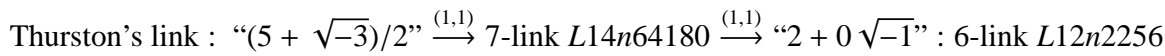
$\stackrel{(1,1)}{\longrightarrow}$ “2 $+0 \sqrt{-3}$ ” : 5-link $L 10 n 113 \stackrel{(1,1)}{\longrightarrow} 4$-link $L 8 n 7 \stackrel{(-1,1)}{\longrightarrow}$ “ $(1+\sqrt{-7}) / 2$ ” : magic 3-link $L 6 a 5$ $\stackrel{(1,1)}{\longrightarrow}$ Whitehead link $L 5 a 1 \stackrel{(1,1)}{\longrightarrow}$ figure-eight knot $K 4 a 1 \stackrel{(1,1)}{\longrightarrow}$ Poincaré homology sphere. 


\begin{tabular}{|c|c|c|c|c|c|}
\hline source & index & cusps & homology & names or $\eta_{d}(M)$ & uqc \\
\hline L6a1 & 3 & 3 & irr: $\frac{1}{2}+\frac{1}{2}$ & L12n2181 & Hesse SIC \\
\hline . & 4 & 5 & irr: $1^{+5}$ & L14n63905 & 2QB MIC \\
\hline Bergé L6a2 & 4 & 4 & irr: $1^{+4}$ & otet16 00025 & 2QB MIC \\
\hline . & 5 & 4 & irr: $1^{+4}$ & otet $20_{00543} ;$ otet $20_{00041} ;$ otet $20_{00549}$ & 5-dit MIC \\
\hline . & 5 & 2 & irr: $\frac{1}{2}+1^{+4}$ & otet $20_{00574}$ & \\
\hline . & 5 & 2 & irr: $\frac{1}{3}^{+2}+1^{+2}$ & otet $20_{00665}$ & \\
\hline magic L6a5 & 3 & 5 & irr: $1^{+5}$ & L14n63788 & Hesse SIC \\
\hline 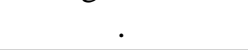 & 4 & 4 & irr: $\frac{1}{2}+1^{+4}$ & $\{31,174,4324,82357, \cdots\}$ & 2QB MIC \\
\hline Borr. L6a4 & 3 & 4 & irr: $1^{+4}$ & ooct06 00466 & Hesse SIC \\
\hline . & 3 & 4 & irr: $1^{+5}$ & ooct $06_{00398}$ & \\
\hline . & 4 & 4 & irr: $\frac{1}{2}^{+2}+1^{+4}$ & $\{63,300,10747, \cdots\}$ & 2QB MIC \\
\hline . & 4 & 6 & irr: $\frac{1}{2}+1^{+6}$ & $\{127,2871,478956, \cdots\}$ & \\
\hline 4-link L8n7 & 3 & 6 & irr: $1^{+6}$ & ooct06 $_{00340}$ & Hesse SIC \\
\hline 5-link L10n113 & 3 & 8 & irr: $1^{+8}$ & 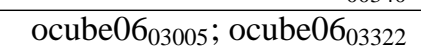 & Hesse SIC \\
\hline 6-link L12n2256 & 3 & 9 & irr: $1^{+9}$ & $\{511,20122, \cdots\}$ & Hesse SIC \\
\hline
\end{tabular}

Table 2. A few 3-manifolds (in column 5) occurring as a model of uqc (in column 6). When not identified in SnapPy the cardinality structure $\eta_{d}(M)$ of finite index subgroups of the 3-manifold $M$ is made explicit. The third column identifies the first homology type, with 1 meaning the ring of integers $\mathbb{Z}$. The 3-manifolds occuring as a model of uqc with source the trefoil knot $\mathrm{K} 3 \mathrm{a} 1=3_{1}$, the figure-eight knot K4a1 and the Whitehead link L5a1 have been investigated in [3] and thus are not included in the table.

and is pictured in Fig. 3. Applying (1,1)-slope Dehn filling on the figure-eight knot, the sequence terminates to the spherical manifold $M_{0}$ that is the Poincaré homology sphere (also known as Poincaré dodecahedral space) $[5,6]$.

Many of the links of the sequence correspond to 'congruence' manifolds [19]. A Bianchi subgroup $\Gamma_{B}$ of $P S L\left(2, O_{d}\right)$ is called a 'congruence' subgroup if there exists an ideal $I \subset O_{d}$ so that $\Gamma_{B}$ contains the 'principal congruence group' $\Gamma(I)=\operatorname{ker}\left\{P S L\left(2, O_{d}\right) \rightarrow P S L\left(2, O_{d} / I\right)\right\}$. Details about these links are available on-line at [20]. Of course congruence Bianchi subgroups generalize congruence subgroups that played a leading role in our approach of uqc [2].

The eight links under scrutiny are pictured in Fig. 3. Four of them are congruence links and are identified by a pair $(-k, I)$ meaning that they act on $\mathbb{Q}(\sqrt{-k}$ with the ideal $I$ : the Thurston's link $(-3,\langle(5+\sqrt{-3}) / 2\rangle)$, the 6-link L12n2256: $(-1,\langle 2+0 \sqrt{-1}\rangle)$, the 5-link L10n113: $(-3,\langle 2+0 \sqrt{-3}\rangle)$ and the magic link L6a5 : $(-1,\langle(1+\sqrt{-7}) / 2\rangle)$. The link $L 10 n 113$ is also otet $10_{00027}$ and the link $L 12 n 2256$ is ooct $04_{00042}$.

The 3-manifolds that we could identify as connected to MICs are in Table 2. This becomes a cumbersome task for links larger than the 6-link. For the link $L 8 n 7$ the cardinality sequence is $\eta_{d}=\{63,794,23753,280162, \cdots\}$, for the link L10n113 it is $\eta_{d}=$ $\{31,176,1987,7628,11682 \cdots\}$ and for the 6 -link it is $\eta_{d}=\{63,580,12243,94274 \cdots\}$. For the latter link, SnapPy is able to build the Dirichlet domain (not shown) of symmetry group $D_{6}$, corresponding to the Hesse SIC while it is not the case for the Hesse SICs attached to the 5-link.

We expect that the full sequence should have a meaning in the context of uqc. 


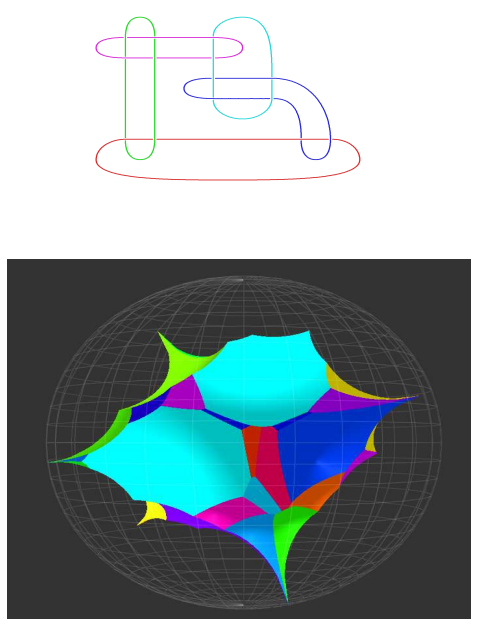

(a)

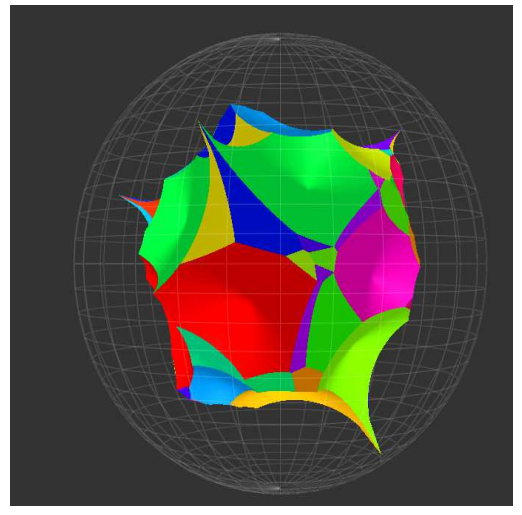

(b)

Figure 2. (a) The 3-manifold $L 14 n 63788$ corresponding to the 3 -fold covering of $L 6 a 5$ relevant to qutrit universal quantum computing, (b) The 3-manifold corresponding to the 4-fold covering of L6a5 relevant to two-qubit universal quantum computing.

\section{Conclusion}

It is not yet known which vista a practical quantum computer will follow. The authors of [3] developed a view of universal quantum computing based on 3-manifolds. In a nutshell, there exists a connection between the Poincaré conjecture -it states that every simply connected closed 3-manifold is homeomorphic to the 3-sphere- and the Bloch sphere $S_{3}$ that houses the qubits. According to this approach, the dressing of qubits in $S_{3}$ leads to 3-manifolds (that have been investigated in much details by W. P. Thurston and led a proof of the Poincaré conjecture) many of them corresponding to MICs (minimal informationally complete POVMs) and the related uqc (universal quantum computing). In [2], uqc, was based on the modular group $\Gamma$ that corresponds to the trefoil knot manifold in [3]. In the present paper, the 3manifolds under investigation derive from subgroups of Bianchi groups, a generalization of $\Gamma$. There seems to exist a nice connection between some congruence subgroups of Bianchi groups through the chain (1) and quantum computing that the author started to investigate. At a more practical level, 3-manifolds can also be seen as (three-dimensional) quasiparticles beyond anyons [22]. 


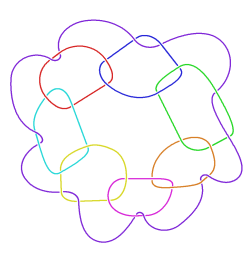

(a) Thurston's link

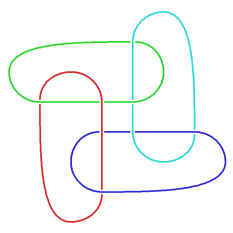

(e) 4-link L8n7

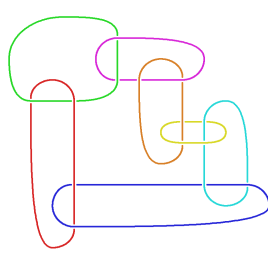

(b) 7-link L14n64180

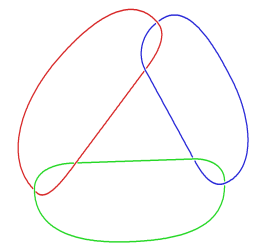

(f) magic 3-link L6a5

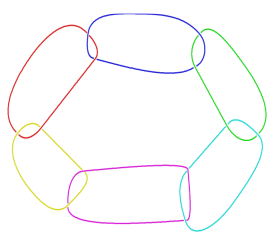

(c) 6-link L12n2256

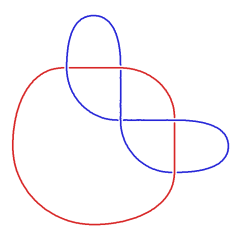

(g) Whitehead link L5a1

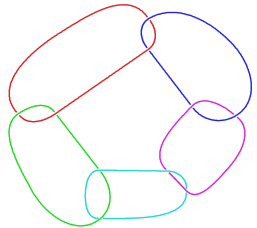

(d) 5-link L10n113

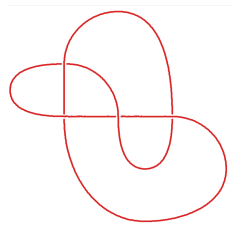

(h) figure-eight knot $\mathrm{K} 4 \mathrm{a} 1$

Figure 3. The consecutive links from (a) to (h) obtained from $(1,1)$ Dehn filling at a single cusp [except for $(-1,1)$ filling at (e)]. The links (a), (c), (d), (f) are congruence. The sequence (d) to (h) already appears in [18].

\section{References}

[1] M. Planat and Z. Gedik, Magic informationally complete POVMs with permutations, $R$. Soc. open sci. 4170387 (2017).

[2] M. Planat, The Poincaré half-plane for informationally complete POVMs, Entropy 2016 (2018).

[3] M. Planat, R. Aschheim, M. M. Amaral and K. Irwin, arXiv 1802.04196 (quant-ph).

[4] M. Planat, R. Aschheim, M. M. Amaral and K. Irwin, arXiv1808.06831 (quant-ph).

[5] C. Maclachlan and A. M. Reid, The arithmetic of hyperbolic 3-manifolds (Springer, New York, 2002).

[6] W. P. Thurston, Three-dimensional geometry and topology (vol. 1), (Princeton University Press, Princeton, 1997).

[7] C. C. Adams, The knot book, An elementary introduction to the mathematical theory of knots (W. H. Freeman and Co, New York, 1994).

[8] M. D. Baker and A. W. Reid, Congruence link complements- a 3-dimensional Rademacher conjecture, Proc. of the 66th Birthday Conference for Joachim Schwermer (2016).

[9] M. Görner, Visualizing Regular Tesselations: Principal Congruence Links and Equivariant Morphisms from Surfaces to 3-Manifolds, Thesis (2011), available online at https://scholar.google.fr.

[10] M. Planat and R. Ul Haq, The magic of universal quantum computing with permutations, Advances in mathematical physics 217, ID 5287862 (2017) 9 pp. 
[11] Chris A. Fuchs, On the quantumness of a Hibert space, Quant. Inf. Comp. 4 467-478 (2004).

[12] M. Appleby, T. Y. Chien, S. Flammia and S. Waldron, Constructing exact symmetric informationally complete measurements from numerical solutions, Preprint 1703.05981 [quant-ph].

[13] F. Grunewald and J. Schwermer, Subgroups of Bianchi groups and arithmetic quotients of hyperbolic 3-space, Trans. Amer. Math. Soc. 335 47-78 (1993).

[14] W. Bosma, J. J. Cannon, C. Fieker, A. Steel (eds), Handbook of Magma functions, Edition 2.23 (2017), 5914 pp.

[15] M. Culler, N. M. Dunfield, M. Goerner, and J. R. Weeks, SnapPy, a computer program for studying the geometry and topology of 3-manifolds, http://snappy.computop.org.

[16] A. D. Mednykh, A new method for counting coverings over manifold with finitely generated fundamental group, Dokl. Math. 74 498-502 (2006).

[17] C. M. Gordon, Dehn filling: a survey, Knot Theory, Banach Center Publ. 42 129-144, Polish Acad. Sci., Warsaw (1998).

[18] B. Martelli, C. Petronio and F. Roukema, Exceptional Dehn surgery on the minimally twisted five-chain link, Comm. Anal. Geom. 22 689-735 (2014).

[19] M. D. Baker, M. Goerner and A. W. Reid, All principal congruence link groups, arXiv 1802.01275 [math.GT].

[20] http://www.unhyperbolic.org/prinCong/

[21] C. Pommerenke and M. Toro, Free subgroups of the parametrized modular group, Rev. Colomb. Matem. 49 269-279 (2015).

[22] S. Vijay and L. Fu, A generalization of non-abelian anyons in three dimensions, arXiv 1706.07070 (2017). 